\title{
Methods of measuring intracranial pressure via the fontanelle without puncture
}

\author{
S. R. WEALTHALL ${ }^{1}$ AND R. SMALLWOOD \\ From the Department of Child Health, University of Sheffield, \\ and the Regional Medical Physics Department, Sheffield
}

SUMMARY It is suggested that non-invasive techniques for measuring intracranial pressure should be suitable for use in the unsedated infant and should be capable of measuring pressure continuously. Methods described by other authors are reviewed. After investigation of 18 patients the technique relying upon the pulsation of the fontanelle described by Purin was rejected as being difficult to perform and applicable only in certain patients with large fontanelles. The technique of using a modified Schiotz tonometer was examined but rejected on grounds of inaccuracy, the need to perform the measurement with the infant in a vertical position, and the varying compressibility of the fontanelle. A method for indirectly estimating intracranial pressure using a modified aplanation principle is described, and a comparison of the pressures so measured and needle pressures is reported The possible uses of a 'fontanometer' using the aplanation principle are discussed and a preliminar report given of its use to monitor the changes of intracranial pressure caused by drugs.

Brett (1966) has pointed out the advantages of measuring intracranial pressure without recourse to puncture of the central nervous system. In addition to avoiding the complications of infection, haemorrhage, or local damage which may result from needle puncture, any such method would allow the repeated or continuous estimation of intracranial pressure. The need for such estimations has been indicated by Lundberg (1960) who showed that, even in the absence of intracranial pathology, long and short-term pressure variations may occur. Although not all of the variations described in adult patients by Lundberg have been reported in infants, Hayden et al. (1970) have shown that, in infants with hydrocephalus, variations in the order of $100 \%$ may occur which are not related to the changes caused by crying or straining. As, in addition, hydrocephalus in infants is now being treated by drugs (Hayden et al., 1970; Lorber, 1972), there is an increasing need for frequent measurements of intracranial pressure over long periods.

1 Requests for reprints: Dr. S. R. Wealthall, The Nuffield Institute for Medical Research, Headley Way, Oxford.
Although methods for continuously measuring intracranial pressure are available in some neuro $\rightarrow+$ surgical centres, it seems unlikely that intraventricular catheters, subdural or epidural strain gauges, or even telemetric devices could be used to monitor intracranial pressure routinely for long periods in the infant. The first three of these methods create a potential entry site for infection and the fourth would require major surgery for insertion and removal of the device. In addition, the technical problems of calibrating a totally indwelling telemetric device and the methods of energizing it are not completely overcome.

In the infant clinicians have been able to obtain some estimation of intracranial pressure by palpation of the anterior fontanelle. Individuals may become skilled at estimating day to day changes in pressure but it is almost impossible to quantitate this subjective assessment. Previous attempts to measure intracranial pressure via the open fontanelle of the infant have been reported (vide infra) but none has passed into general usage. The aim of this study was to examine simple techniques of measuring intra88 
cranial pressure which make use of the anterior fontanelle and which could be used in the unsedated, unshaven infant.

\section{METHODS RELYING ON PULSATION OF ANTERIOR FONTANELLE}

Riechert and Heines (1950) measured the pulsation amplitude in a tambour applied to the dura mater. As the pressure in the tambour was increased, the pulsation amplitude also increased and seemed to reach a maximum. It was suggested that the tambour pressure which gave the maximum amplitude of pulsation related to the intracranial pressure. Purin (1964) extended Riechert and Heines' (1950) work and, in a small number of patients, showed that as the pressure applied to the anterior fontanelle was increased the pulsation amplitude in the tambour increased and then declined. He concluded that the peak pulsation amplitude occurred when the pressure within the tambour equalled that within the skull. In 13 infants Purin found that the pressure determined by his method and that found by lumbar or ventricular puncture a few minutes later were almost identical. His findings were confirmed by Barashnev and Leontiev (1966) who find high 'fontanometer' pressures in infants with cerebral injury at birth.

Despite these encouraging findings Purin's method of determining intracranial pressure has not passed into general use, although the method would seem to be very simple. Our study of Purin's technique was performed to investigate its usefulness as a routine clinical procedure.

\section{METHOD}

A $3 \mathrm{~cm}$ diameter latex covered tambour was applied to the fontanelle and held in position by rubber and Velcro straps. The tambour was connected to a $T$ piece, one arm of which led to a pressure transducer and the other to a sphygmomanometer bulb. The output from the transducer was displayed both on a storage oscilloscope and as an average pressure on a moving coil meter. In suitable investigations recordings of the transducer output were made. After allowing the infant to settle in a supine position the pressure in the tambour was raised by means of the sphygmomanometer bulb. The pressure was maintained at a given level until the pulsation displayed on the oscilloscope screen was of a 'constant' amplitude and the applied pressure and pulsation amplitude were recorded. Pressure was raised and lowered several times to obtain pulsation amplitudes at rising and falling pressures. Minor movements of the infant were ignored but crying, struggling, or sucking caused that particular series of readings to be discarded.

CALCULATION OF RESULTS In infants where records of pulsation amplitude were obtained at several applied pressures without interruption the average pulsation amplitude for any applied pressure was calculated. Where sufficiently long recordings were obtainable the standard deviation of the mean pulsation amplitude was also calculated for each applied pressure.

By this means the significances of the differences between mean pulsation amplitudes at different applied pressures could be determined for each patient by Student's $t$ test. The results of this procedure for the only patient showing a peak amplitude of pulsation are shown in the Table.

\section{TABLE}

PULSATION AMPLITUDES AT DIFFERENT APPLIED PRESSURES

\begin{tabular}{|c|c|c|c|}
\hline $\begin{array}{c}\text { Applied } \\
\text { pressure } \\
\text { (mm water) }\end{array}$ & $\begin{array}{c}\text { Mean } \\
\text { pulsation } \\
\text { amplitude } \\
\pm 1 S D \\
\text { (mm water) }\end{array}$ & $\begin{array}{c}\text { Determinations } \\
\text { (no.) }\end{array}$ & $\frac{\text { Difference of means }}{S E \text { of mean differences }}$ \\
\hline $\begin{array}{r}50 \\
100 \\
200 \\
300 \\
400\end{array}$ & $\begin{array}{l}17 \cdot 1 \pm 1 \cdot 2 \\
24 \cdot 3 \pm 3 \cdot 0 \\
32 \cdot 9 \pm 9 \cdot 0 \\
29 \cdot 9 \pm 5 \cdot 4 \\
27 \cdot 0 \pm 4 \cdot 1\end{array}$ & $\left.\begin{array}{r}10 \\
9 \\
10 \\
10 \\
10\end{array}\right\}$ & $\begin{array}{r}21^{*} \\
16^{*} \\
19^{*} \\
9^{*}\end{array}$ \\
\hline
\end{tabular}

* Significant by $t$ tables at $>0.001$.

\section{RESULTS}

The technique was used in 17 unsedated infants and in one infant lightly sedated with chloral hydrate. Records which did not include interruptions due to crying or movement were obtained only in six infants, because of the difficulty of inflating the tambour without disturbing the infant.

VARIATION OF PULSATION AMPLITUDE Gross body movements, crying, or sucking resulted in large changes in the pulsation amplitude recorded from the tambour. Changes in pulsation ampli- 


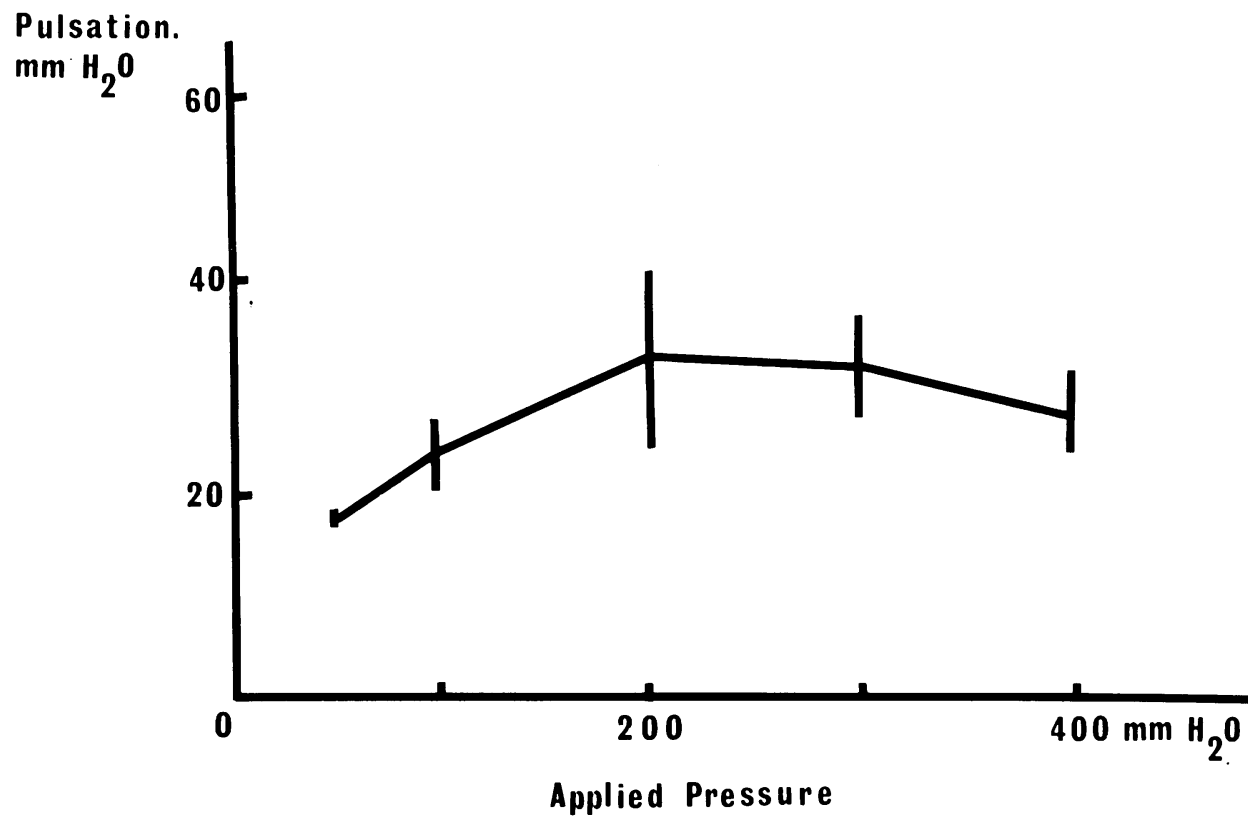

FIG. 1. Relationship between applied pressure and pulsation amplitude. Of six patients analysed in detail on this study only one showed the peak of pulsation amplitude described by Purin. He had a very large fontanelle. Vertical bars represent $1 S D$.

tude were also noted in the absence of any body movement or change in state of consciousness, respiration, or pulse. The variation of pulsation amplitude was studied in detail in one patient who had been sedated and in whom the applied pressure was maintained at a constant level for periods of five minutes. Although this patient remained completely undisturbed during the investigation, the pulsation amplitude at constant pressure varied by up to $30 \%$ of the mean applied pressure. This variation was of the same magnitude as that produced by varying the applied pressure over $400 \mathrm{~mm}$ of water. Thus the spontaneous variation of pulsation amplitude over a few minutes is of the same size as the variations produced by different applied pressures.

RELATION OF APPLIED PRESSURE TO PULSATION AMPLITUDE The spontaneous variation in pulsation amplitude made identification of a meaningful pulsation amplitude at any applied pressure difficult, but in six patients sufficient results were obtained to allow calculation of valid mean pulsation amplitudes and graphing of pulsation amplitude against applied pressure. In only one of the six patients (Fig. 1) was the peak of pulsation pressure described by Purin found. The other five patients showed no evidence of either a peak pulsation amplitude nor the maximum described by Reichert and Heines. All six patients had needle pressures measured on the day of the investigation. Their manometric pressures were within the range of applied pressures used. In the one patient showing a peak the 'fontanometer' pressure and the manometric pressure were in agreement.

\section{DISCUSSION}

Failure to obtain sufficient information for statistical analysis in two-thirds of the patients studied, despite choosing periods when the infant was recently fed and sleepy, suggests that the procedure is difficult to perform without disturbing the infant. As one of the prime requisities for a 'fontanometer' is that it can be used under normal conditions and at rest it was decided that 
sedation of infants for the investigation would not be justifiable for routine use.

The spontaneous variation in pulsation amplitude encountered in all patients presumably reflects changes in intracranial pulse pressure. Alterations in pulse pressure may be caused by changes in arterial pressure (Sibayan et al., 1970), cranial venous pressure (Guthrie et al., 1970), cerebrospinal fluid (CSF) pressure (Davson, 1970), and the unknown mechanisms which control the pulsation absorbing and damping system of the spinal theca and veins (Martins et al., 1972). By averaging pulsation pressures over several five second periods it was possible to identify significant changes in pulsation amplitude between different applied pressures. The time interval for averaging pulsation amplitudes is critical; if too short, there will be errors due to the spontaneous variations described; if too long, there will be errors due to the rapid variations of cerebrospinal fluid pressure already referred to. The difficulty of balancing these conflicting requirements is one of the major problems of Purin's technique.

The identification of a peak pulsation amplitude in only one patient analysed in detail may be explained by the large size of the fontanelle in this exceptional patient, a 9 month old infant with a head circumference of $64 \mathrm{~cm}$ caused by untreated hydrocephalus who had an anterior fontanelle measuring $9 \mathrm{~cm} \times 7 \mathrm{~cm}$. All other infants studied had fontanelles which had the majority of their surface covered by the tambour. Experiments with models have suggested that when a tambour occupies a large proportion of the fontanelle the pressure increase in the tambour results in an increase of intracranial pressure. Any increase in intracranial pressure is liable to produce an increased pulsation pressure within the skull which will only stop when autoregulation of cerebral blood flow is lost at very high intracranial pressures (Hussey et al., 1970). Thus in infants with normal to moderately enlarged fontanelles the application of a tambour of comparable size and its inflation is likely to lead to an increase of pulsation amplitude within the physiological pressure range. The results in the five patients in whom no peak of pulsation amplitude was demonstrated may be explained by this process. The findings in the exceptional patient may be explained by the large fontanelle size, as in this situation intracranial pressure will not be increased by inflation of the tambour.

Attempts to reduce the size of the tambour in relation to the fontanelle have failed due to the small amplitude of pulsations produced from smaller systems and the difficulty of applying the small tambour to the fontanelle without causing pressure to be transmitted to the brain.

Thus it would seem that, although in exceptional patients with large fontanelles Purin's technique may provide a measure of intracranial pressure, it is difficult to apply in the majority of patients. In addition the large number of determinations and calculations required to obtain valid results renders the technique as described unsuitable for routine use. The method may, however, provide some information on the ability of the craniospinal axis to deal with increases in pressure in a manner similar to that used by Katzman and Hussey (1970) who raised intracranial pressure by infusing fluid into the CSF system and measuring the pressure responses.

\section{METHODS RELYING ON TENSION OF ANTERIOR FONTANELLE}

The tension of a membrane containing a fluid is determined by the pressure of the contained fluid and the elasticity of the membrane. Under conditions of constant elasticity changes of pressure will therefore be reflected by changes in membrane tension. This principle is used in ophthalmic instruments used for measuring intraocular pressure such as the Schiotz tonometer. In this instrument the amount of depression of the cornea by a weighted plunger provides a measure of corneal tension. By means of a lever system and the use of standard weights this measurement of corneal tension can be translated into intraocular pressure. The use of this system requires an assumption that corneal elasticity is constant or nearly so. This may be true for the cornea but it has not been shown to be so for the membranes which make up the anterior fontanelle. Thus, techniques for estimating intracranial pressure using the modified Schiotz tonometer are difficult to evaluate, although Davidoff and Chamlin (1959) and others have reported its use for this purpose. With the additional limitations imposed by having to perform all measurements with the child in a sitting position to allow gravity 
to act on the plunger, the method would appear unsuitable for routine or continuous measurement of intracranial pressure.

As the elasticity of the fontanelle may vary from time to time and from child to child, a method not relying on the elasticity of the membrane is preferable. Such a technique is provided by the aplanation principle.

If a plane surface (guard ring or baseplate) is placed against a membrane distended by pressure the membrane will bulge through any hole in the surface. If a plunger is placed in the hole and a force applied to it so that the end of the plunger is in the same plane as the baseplate, the pressure exerted by the plunger will be equal to that within the membrane. In these circumstances the membrane is flat and so there is no effect due to tension in the membrane nor any effect due to compression of the membrane. Thus the method should be independent of membrane characteristics.

USE OF APLANATION TRANSDUCER The APT-16 transducer (Hewlett Packard) uses a modification of the aplanation principle and was evaluated for use as a 'fontanometer'. The plunger in this transducer is spring loaded, so that the membrane bulges through the hole in the baseplate until the force exerted by the spring is equal to the pressure within the membrane. As the plunger movement is $0.0011 \mathrm{~mm}$ for an applied pressure of $1 \mathrm{~mm}$ mercury, the effect of tension in the membrane can be ignored. The movement of the plunger is measured by a differential transformer technique. To evaluate the performance of this technique, measurements of pressure by needle and by aplanation transducer were performed simultaneously in a small number of infants.

\section{METHOD}

An ileostomy flange (internal diameter $30 \mathrm{~mm}$ ) was applied to the scalp over the fontanelle by means of adhesive. The aplanation transducer was secured into the flange by means of a 'jubilee' clip and the infant allowed to settle, after being placed in a special frame to support the head in a horizontal position The lateral angle of the fontanelle was prepared foe ventricular puncture (all infants studied had large $\underset{ }{-}$ fontanelles) and a routine puncture performed. The needle was connected to an autoclaved pressure transducer (Bell and Howell type L221) by sterile

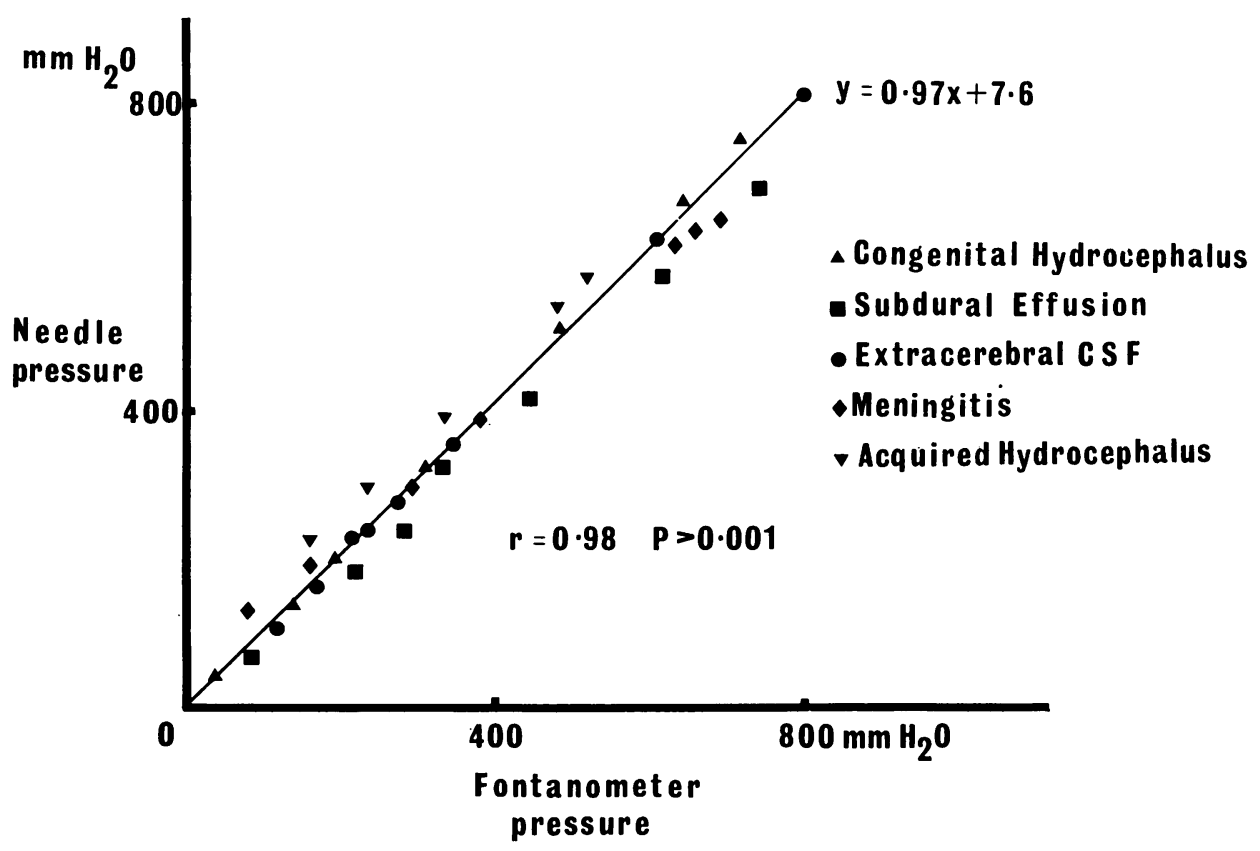

FIG. 2. Relationship between intracranial pressure measured by needle and aplanation transducer (fontanometer). Results from five patients and their conditions are shown. 


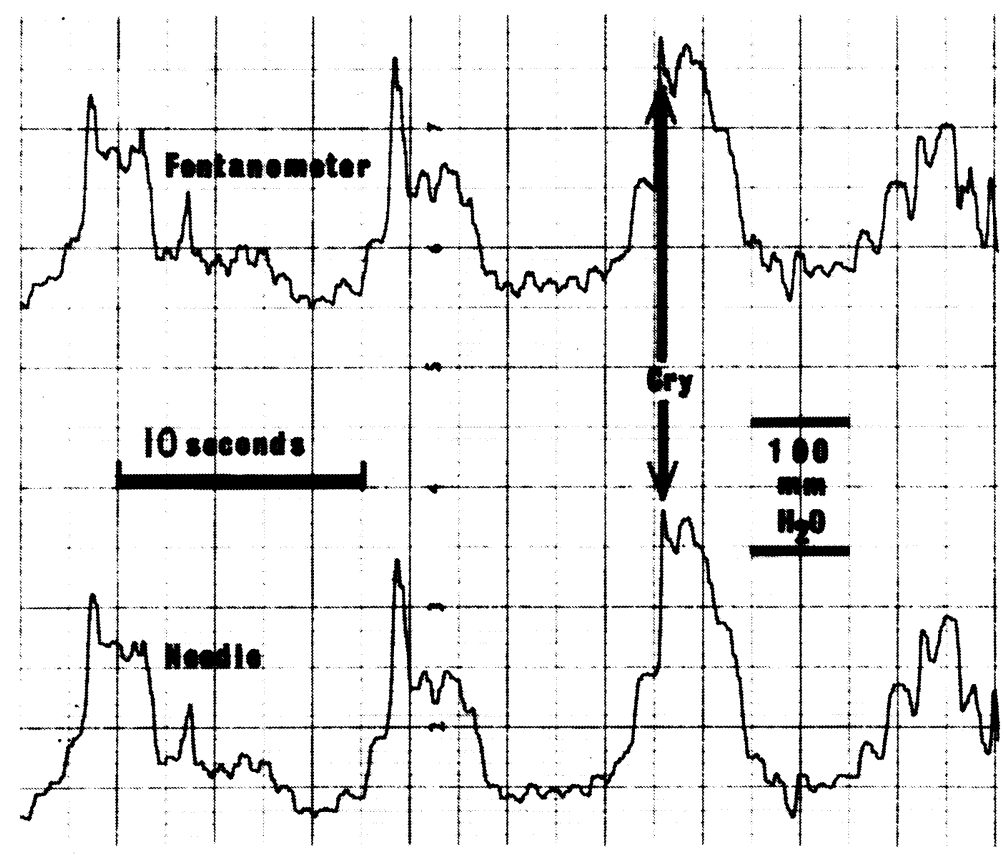

FIG. 3. Simultaneous recordings of needle and aplanation transducer pressures. The child is sobbing periodically.

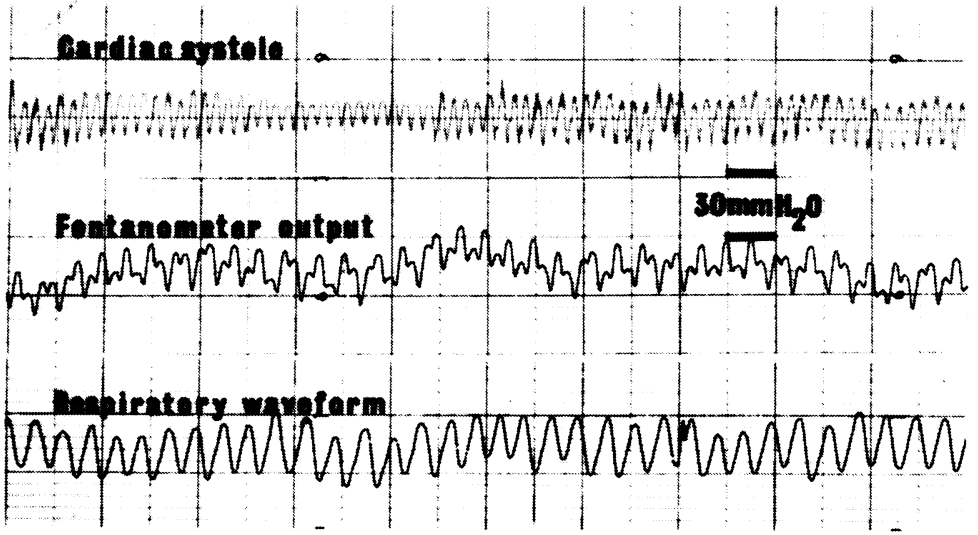

FIG. 4. Simultaneous recording of cardiac systole, fontanometer output, and respiratory movements. The large regular peaks of pressure coincide with respiratory movements and the smaller peaks with cardiac systole.

plastic tubing and the output of the pressure transducer and aplanation transducer recorded by means of a potentiometric pen recorder. Recording continued until the investigation, for which the ventricular tap was performed, was complete.

\section{RESULTS}

Five infants were investigated in this manner.
Their clinical conditions are shown in Fig. 2. The variations of intracranial pressure caused by cardiac systole, respiration, and sobbing were displayed equally well by pressure transducer and aplanation transducer (Fig. 3). A correlation between pressures measured by the two methods in five patients (Fig. 2) indicates that the aplanation transducer faithfully measured intracranial 


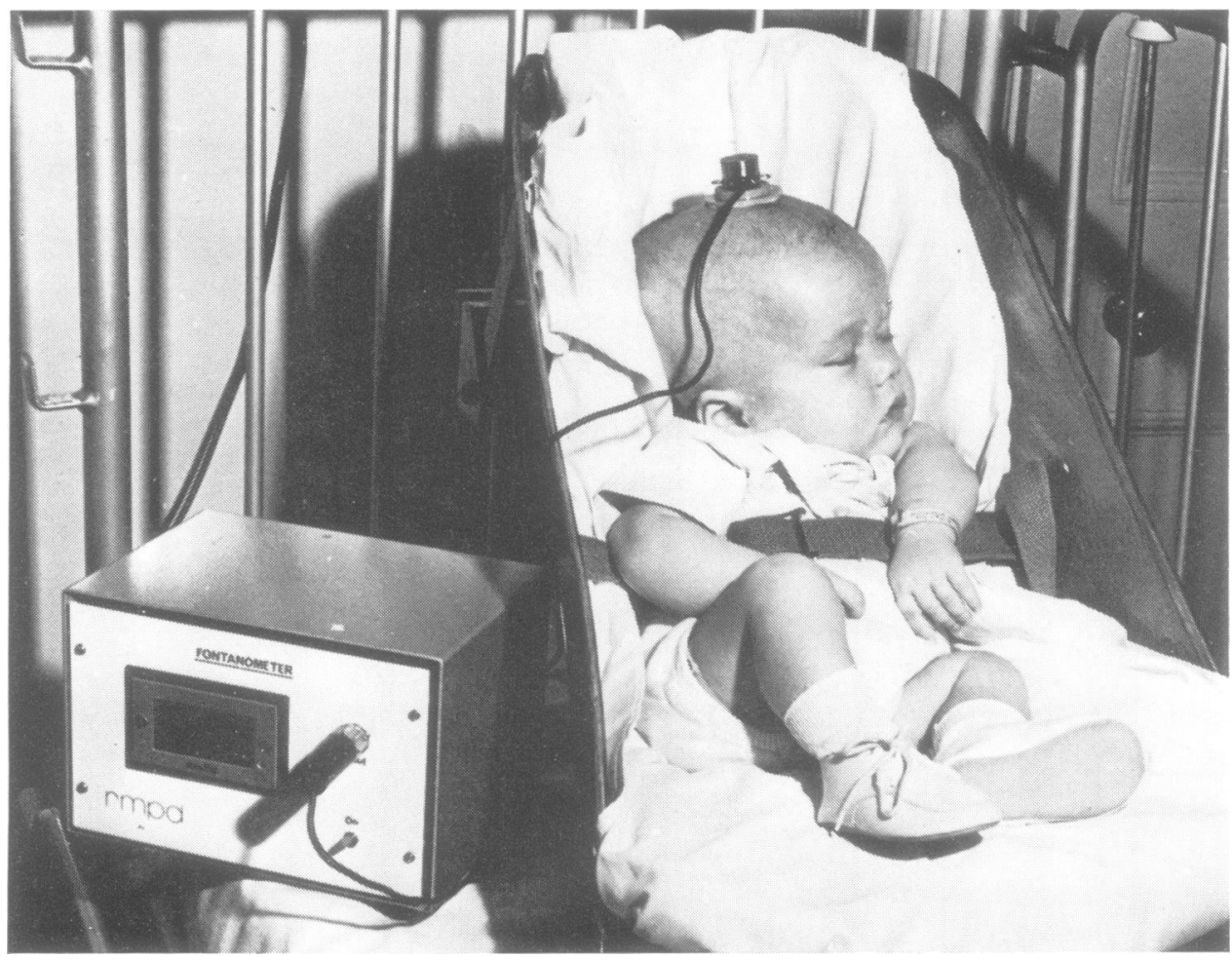

FIG. 5. Use of aplanation transducer for continuous measurement of intracranial pressure.

pressure over a wide range in each patient. At higher gain levels the aplanation transducer was sufficiently sensitive to display pressure variations due to cardiac systole and respiration and to allow their measurement (Fig. 4).

\section{DISCUSSION}

The results indicate that the aplanation principle as used by the Hewlett Packard transducer may be used to measure intracranial pressure without puncture in infants with a variety of conditions. It is obvious that the fontanelle must be of sufficient size for the circumference of the hole in the baseplate to be in contact with the fontanelle and not with its bony edge. Likewise the fontanelle must protrude above the bony margins. The position of the child is critical in using the aplanation transducer as the latter is measuring pressure at the level of the fontanelle and so allowance must be made for the distance between fontanelle and any chosen reference point such as the right atrium or lowest point of the head. Such an allowance can be added automatically by providing an offset control on the power supply and display unit. The attitude of the transducer in relation to gravity is also important for accurate measurement. The transducer should always be 'zeroed' in the attitude in which it is to be used. Thus, if the infant is to lie supine and the transducer is to be applied to the fontanelle with its baseplate in a vertical attitude, it should be 'zeroed' in this attitude.

USES OF APLANATION TECHNIQUE Application of the transducer to the fontanelle in an ileostomy flange was found to be useful for making continuous measurements for long periods. The infant may be placed in a tilting chair to facilitate nursing and, as no puncture is to be performed, the flange may be sited centrally over the fontanelle, so causing less problem with the tilting of the transducer (Fig. 5). In this position, it is important that the cable of the transducer be 


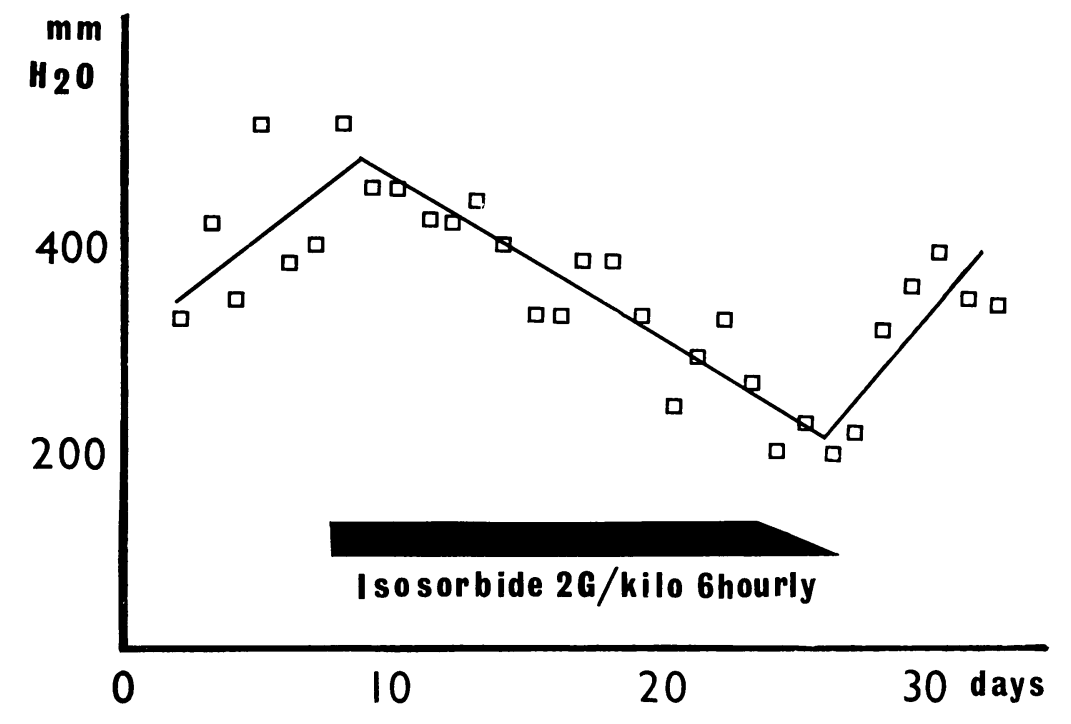

supported and fixed in such a manner that turning movements caused by the infant are not transmitted to the transducer as the edges of the hole in the baseplate may then lose contact with the scalp, so invalidating measurements. The device may also be used for intermittent measurements of intracranial pressure-for example, to monitor the effects of drugs (Fig. 6) - in infants with raised intracranial pressure. In small infants the best results are obtained when held in a horizontal position in the arms of a nurse. The transducer is then applied to the fontanelle with its baseplate vertical until a 'constant' measurement is obtained. Only sufficient pressure to bring the periphery of the hole into contact with the scalp is required and excessive pressure can be avoided by 'buttressing' the device against the scalp with the fingers that are holding it. In this use the output of the transducer may be displayed directly on a digital voltmeter in millimetres of mercury or as a recording on a pen recorder.

\section{CONCLUSIONS}

In our hands, Purin's method of estimating maximal pulsation amplitude of the fontanelle has been unsuitable for routine clinical measurement of intracranial pressure. Methods using modifications of the Schiotz tonometer would not seem capable, either theoretically or practically, of providing reliable or continuous measurements of intracranial pressure. The modified aplanation principle as used by the Hewlett Packard APT 16 transducer would seem to be suitable for the measurement of intracranial pressure without puncture in infants with open fontanelles.

Funds for the purchase of the Hewlett-Packard aplanation transducer were provided by the R.I.C.H.A.R.D. Fund. The patients investigated were under the care of Dr. J. A. Black, Mr. J. Lister, Dr. J. Lorber, and Mr. R. B. Zachary. Technical assistance was provided by the Regional Medical Physics Department and C. Ball, and photography by Mr. A. K. Tunstill.

\section{REFERENCES}

Barashnev, Y. I., Leontiev, A. F. (1966). Cerebrospinal fluid pressure in premature infants with and without intracranial birth injury. (Abstract.) Developmental Medicine and Child Neurology, 8, 117.

Brett, E. M. (1966). Measurement of cerebrospinal fluid pressure in infants without puncture. Developmental Medicine and Child Neurology, 8, 207-210.

Davidoff, L. M., and Chamlin, M. (1959). 'The fontanometer.' Adaptation of the Schiotz tonometer for the determination of intracranial pressure in the neonatal and early periods of infancy. Pediatrics, 24, 1065-1068.

Davson, H. (1967). The Physiology of the Cerebrospinal Fluid. Churchill: London. 
Guthrie, T. C., Dunbar, H. S., and Karpell, B. (1970). Ventricular size and chronic increased intracranial venous pressure in the dog. Journal of Neurosurgery, 33, 407-414.

Hayden, P. W., Shurtleff, D. B., and Foltz, E. L. (1970). Ventricular fluid pressure recordings in hydrocephalic patients. Archives of Neurology, 23, 147-154.

Hussey, F., Schanzer, B., and Katzman, R. (1970). A simple constant-infusion manometric test for measurement of CSF absorption. 2. Clinical studies. Neurology (Minneap.), 20, 665-680.

Katzman, R., and Hussey, F. (1970). A simple constantinfusion manometric test for measurement of CSF absorption. 1. Rationale and method. Neurology (Minneap.), 20, 534-544.

Lorber, J. (1972). The use of isosorbide in the treatment of hydrocephalus. Developmental Medicine and Child Neurology, 14, Suppl. 27, 87-93.
Lundberg, N. (1960). Continuous recording and control of ventricular fluid pressure in neurosurgical practice. Acta Psychiatrica et Neurologica Scandinavica, 36, Suppl. 149.

Martins, A. N., Wiley, J. K., and Myers, P. W. (1972). Dynamics of the cerebrospinal fluid and the spinal dura mater. Journal of Neurology, Neurosurgery, and Psychiatry, 35, 468-473.

Purin, V. R. (1964). Measuring intracranial pressure of a child without the help of puncture. A new method. Pediatriya, 43, No. 5, 82-85.

Riechert, T., and Heines, K.-D. (1950). Über zwei Untersuchungsmethoden zur Beurteilung der Hirndurchblutung. Nervenarzt, 21, 9-16.

Sibayan, R. Q., Begeman, P. C., King, A. I., Gurdjian, E. S., and Thomas, L. M. (1970). Experimental hydrocephalus. Archives of Neurclogy, 23, 165-172. 